\section{EMBRYARIDDLE \\ Aeronautical University}

SCHOLARLY COMMONS
International Journal of Aviation, Aeronautics, and Aerospace

$10-23-2014$

\title{
Improving Airplane Touchdown Control by Utilizing the Adverse Elevator Effect
}

Nihad E. Daidzic Ph.D., Sc.D.

AAR Aerospace Consulting, LLC, aaraerospace@cs.com

Follow this and additional works at: https://commons.erau.edu/ijaaa

Part of the Aeronautical Vehicles Commons, Control Theory Commons, Dynamic Systems Commons, Navigation, Guidance, Control and Dynamics Commons, and the Ordinary Differential Equations and Applied Dynamics Commons

\section{Scholarly Commons Citation}

Daidzic, N. E. (2014). Improving Airplane Touchdown Control by Utilizing the Adverse Elevator Effect. International Journal of Aviation, Aeronautics, and Aerospace, 1(4). https://doi.org/10.15394/

ijaaa.2014.1032

This Article is brought to you for free and open access by the Journals at Scholarly Commons. It has been accepted for inclusion in International Journal of Aviation, Aeronautics, and Aerospace by an authorized administrator of Scholarly Commons. For more information, please contact commons@erau.edu, wolfe309@erau.edu. 
Thousands of landings in commercial transport-category (FAA, 2013) airplanes are executed every single day all over the world. Accurate touchdowns are crucial in saving excessive wear and tear on brakes, tires, and airplane structure. They are especially critical when landing on wet and/or contaminated runways or during LAHSO operations. Despite so much operational experience, the control of landing flare and touchdown accuracy is still a "hit or miss" event. The statistics on touchdown points and the large scatter of data indicates that landings are not very accurate even when full auto-land capabilities are used (FAA, 2007). Large operational cushions (67\% for dry DLDR and 92\% for wet WLDR runway) are provided over (un-factored gross measured) demonstrated dry landing distance (gross DDLD) for airplanes used in commercial air transportation (e.g., Title 14 CFR 121). Contaminated runways may require 2-3 times longer runway lengths than DDLD (Daidzic \& Shrestha, 2008; Daidzic, 2011a). The highest frequency of transport-category airplane incident/accidents occurs indeed during the landing/rollout phase.

In normal daily operations, various studies over many decades have shown the standard deviation (SD) of touchdown points in manual landings to be roughly 1000 feet for any particular transport-category airplane type. This indicates that more than $30 \%$ of touchdowns are actually exceeding even these large uncertainties. A significant percentage of touchdowns occurs beyond the $3,000 \mathrm{ft}$ markers (touchdown zone) which reduces the margin for error when landing on slippery runways. The lowering of the nose gear often takes unnecessarily long time in line operations further reducing the distance margins for subsequent deceleration (Daidzic \& Shresta, 2008). Not every long-landing results in a runway overrun, but in every overrun accident, landing long and having poor touchdown control was a major contributing factor (Daidzic \& Shresta, 2008; Daidzic 2009a, 2009b, 2009c, 2011a, 2013). Contemporary conventional flight control design and pilot training does not address landing touchdown accuracy and control sufficiently well.

The main gear touchdown point depends on many factors, such as, proper vertical flight-path, airspeed, crossing threshold at appropriate height, proper thrust management, height and flight load in flare, atmospheric turbulence and wind, etc. The landing maneuver can be seen as a management of the airplane's total energy. A conventional landing flare maneuver involves pitching airplane slightly up while simultaneously closing the throttles when the gear height is "about right" (Blake and Elliott, 1991; Daidzic, 2011b; Davis, 1971; Denton, 1993; Lowery, 2001; Schiff, 1997; Webb and Walker, 2004). To pitch the airplane up, a pilot must pull elevator up which initially generates unbalanced downward force thereby rotating airplane's nose up and ultimately increasing the 
pitch angle, the Angle-of-Attack (AOA), and reducing the flight path angle (must still be negative for positive touchdown). However, before an airplane starts pitching up, increasing AOA and lift, the initial airplane's reaction will be downward acceleration due to unbalanced tail down lift increment (Phillips, 1979; Grantham et al, 1987). This phenomenon is called Adverse Elevator Effect (AEE). There is nothing peculiar about it as all conventional flight controls initially exhibit adverse responses.

In a cruise flight one is not much concerned about the AEE and small altitude loss (several feet at maximum) before an airplane actually starts climbing. However, in landing approach several feet could mean a difference between a smooth and a hard landing with possible bounce, balloon, extended float, runway overrun, etc. But even worse than this well-known reverse-altitude response is the time-lag (dead-time) introduced by the AEE and the "nothing-happening-whenpulling-elevator" syndrome. As many studies have shown and almost every pilot knows, it is almost impossible to make consistent (manual or automatic) landings and touchdowns. Landing flare maneuver in transport-category airplanes is somewhat mechanical although many would not admit it. Landing flare is indeed of very short duration and thus very difficult to practice and refine.

The abbreviated review of some important publications on landing flare presented here is neither exhaustive nor complete. A whole article would be required just to review all the work done in the past 50+ years on landing dynamics of jet airplane. One of the first studies of landing touchdowns in turboprop and turbojet-type airplanes was conducted by Stickle (1961). The author found that most of the touchdowns occurred at airspeeds of 22.5 to $26.6 \%$ above the stalling speed. He also found that 1-in-100 touchdowns occurred beyond 2,800 ft from the runway threshold in turbojet-type airplanes. White (1968) presented a very simple model of landing flare which did not have the sophistication required to model the AEE. The AEE was known, but not understood or appreciated well enough before the arrival of the large airplanes and the onset of the "jet-age". To the best of our knowledge, the first serious theoretical analysis of landing flare was presented by Pinsker (1969). The author used the Laplace transform on the simplified linear 3-DOF longitudinal airplane dynamics model to obtain the short-term aircraft response after step and ramp elevator control applications. Unfortunately, Pinsker's original work on AEE had some typographical errors in equations used. The author himself was truly a giant of aircraft stability, control, and performance theory who made numerous important contributions to the field and who also provided inspiration to the author of this article. Cleveland (1970) discussed handling qualities of conventional aircraft designs. As Cleveland pointed out, reverse altitude response 
(i.e., AEE) can be accepted if the longitudinal time-lag of response does not exceed 2 seconds criterion. As an example Cleveland used C-5A with a dead-time in pitch response of about $0.8 \mathrm{~s}$. The conclusion made is that future very large aircraft may indeed need canard surface and perhaps Direct Lift Control (DLC) due to unacceptable dead-time in pitch response. Lykken and Shah (1972) discussed the use of DLC to improve the pitch response lag times and better vertical response control in large airplanes (Lockheed's L-1011). This was especially important for the precise glideslope control and tracking during Category III ILS approaches where almost instantaneous lift management is critical. Seckel (1975) presented his study of the landing flare in light GA airplane (variable-stability Navion) and did not mention AEE as relevant to landing flare. Phillips (1979) presented his in-depth analysis of altitude response based on the impulse, step and ramp elevator application. The author used the case of Space Shuttle orbiter and other four (military) aircraft of which some were used in power-off landing scenarios to investigate approach and landing dynamics. The author used 3-DOF linearized equations of longitudinal aircraft dynamics. His results predicted altitude loss and time-delay of proverse flight-controls effect of 1-2 seconds. Phillips made no connection between the reverse altitude response and the landing flare. Grantham et al (1987) performed full 6-DOF simulation and piloted study of handling qualities in pitch response lag for a large transportcategory airplane (Lockheed's L-1011). Abzug and Larrabee (1997) discussed, among many other things, the handling properties and reverse altitude response of very large aircraft during landing flare. The authors merely repeated the arguments already given by Cleveland (1970) and Phillips (1979) and were using STS's space shuttle orbiter's sluggish and inadequate longitudinal dynamic and time-lags in pitch response that exceeds 2 seconds. Merat (2008) considered the use of DLC in Airbus 380 due to its anticipated sluggish longitudinal dynamics in landing. Merat showed the existence of the reverse altitude response and the way to mitigate it using the DLC. Merat employed the standard small-perturbation 3DOF state space analysis of longitudinal dynamics. It may still be too early to determine if A380 may indeed need DLC in the future. Zaal et al (2008) studied the effect of pitch and heave motion cues in a pitch control task. Detecting AEE accurately may be critical in our ability to use it for our advantage. The issue of AEE was revisited in publications by Daidzic (2010, 2011b), where for the first time it was suggested to be used to pilot's advantage during landing flare and a new landing technique for large airplanes has been proposed. Very recently Malmquist et al (2014) presented their study of kinematic effects in large transport aircraft mentioning the reverse altitude response. Authors also recommended incorporating training concerning kinematic issues for operators of large aircraft. 
Historically, very little "science" existed in pilot education, training and execution of the landing flare maneuver. This led to large scatter in touchdown points and much too frequently a runway overrun (Daidzic, 2009b, 2009c, 2011a, 2011c). Typically, an airplane travels horizontally about 200-250 feet every second in flare maneuver. Any prolonged float, bounce, and/or ballooning uses runway excessively and makes overrun or subsequent heavy breaking more probable and dramatic.

An illustration of landing flare (round-out) maneuver is shown in Figure 1. It is assumed that a constant flight load is maintained during flare curve which then represents a segment of a circle. One has to be very careful with the definition of the flare height which is not the same as a runway threshold crossing height (TCH). Any horizontal distance covered in actual flare must be added to the distance covered to descend from the TCH to flare height (if lower). Proper flare height and accompanied horizontal flare distance can theoretically be zero if no-flare touchdown is made. Once the airplane's gear height achieves "appropriate" flare height, the pilot starts the pull-up maneuver while simultaneously closing the throttles. So much latitude exists here as various flare heights with different pitch-up load profiles of various durations are used. The purpose of a good landing flare is to progressively pitch up an airplane to a proper touchdown attitude and arresting the sink rate, while simultaneously avoiding bounce, float, and/or ballooning. This is by no means a simple task as the maneuver is very short and the human and airplane inertia makes the feedback process complicated and sluggish at best.

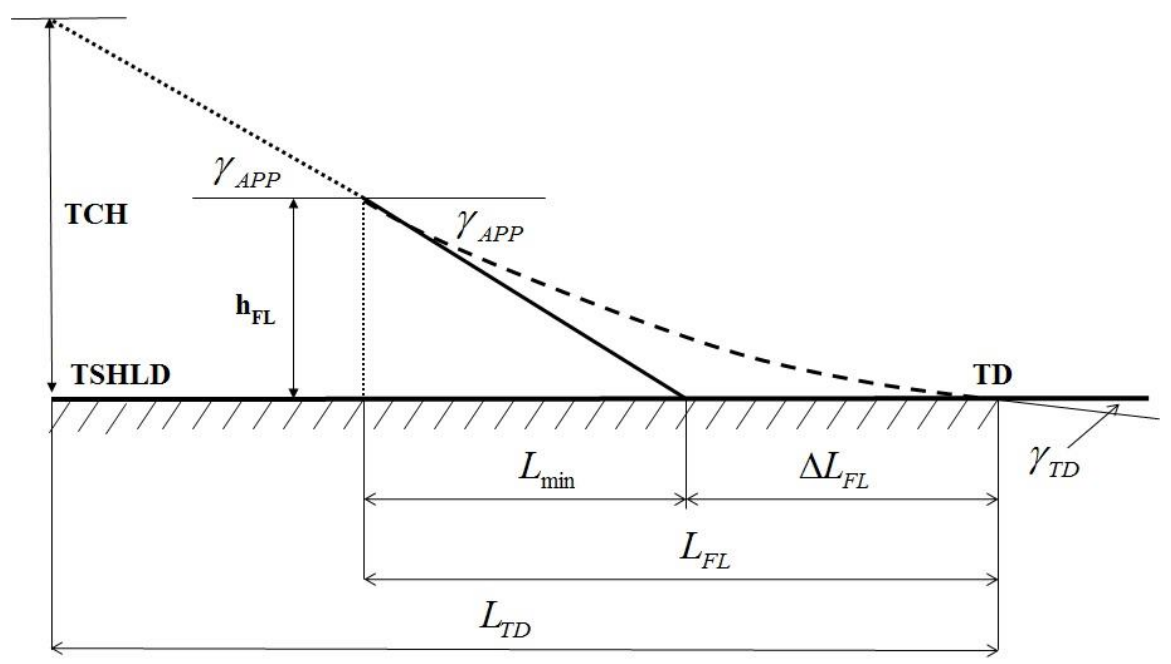

Figure 1. Flare maneuver. Not to scale. Angles and distances are highly exaggerated for better visual representation. 
The rounding out maneuver can be executed using infinite combinations of flight load profiles of different durations. Often, the pilot is interested in gentle rounding-out maneuver which offers more time to monitor progress for "normal" touchdowns. However, gentle flare will result in touching down long and wasting usable runway. If the runway is contaminated and/or LAHSO operations are in use, this could cause serious problems in subsequent ground-roll braking efforts, as well-documented accidents history has unfortunately shown. An additional horizontal distance will be covered in curved path beyond the point of straight descent (glidepath) and runway intercept as shown in Figure 1. The operational practice of many operators uses fixed flare height (e.g., 30-ft for wide-body and $15-20 \mathrm{ft}$ for narrow-body) which pilots are then instructed to follow. There is rarely any compensation for variable conditions and everything is left to the pilot's experience of how to flare and control touchdown.

The flare height can be estimated from Daidzic \& Shrestha (2008), by assuming constant (or averaged) instantaneous flare flight-load:

$h_{F L}=\frac{v_{F L}^{2} \cdot \varsigma_{F L}^{2}}{2 \cdot g \cdot\left(n_{F L}-1\right)} \cdot\left(\gamma_{A P P}^{2}-\gamma_{T D}^{2}\right)=\frac{R O D_{A P P}^{2}-R O D_{T D}^{2}}{2 g \cdot \Delta n_{F L}}$

Where:

$\varsigma_{F L}=\left(1 \pm \frac{v_{w}}{v_{T A S}}\right) \quad v_{F L G S}=v_{F L} \cdot \varsigma_{F L} \quad \Delta n_{F L}=n_{F L}-1$

The "proper" flare heights versus approach rate-of-descent (ROD) and various flight loads for touchdown vertical of 200 and $100 \mathrm{fpm}$ respectively using Equation 1 is shown in Figures 2 and 3. Since the normal landing reference airspeed is $30 \%$ over the stall speed in a given configuration, the maximum vertical flight load allowed is 1.69 which is depicted as dashed line. Similar results are shown in Figure 4, only this time for the zero-sink or tangential ("greaser") touchdown. If the touchdown angle is the same as the approach angle the "proper" flare height is obviously zero. While instantaneous application of constant flare load was assumed here, Pinsker (1969) considered time evolution of pitch response.

Computations of the flare height as a function of approach ROD (just before flare starts), pull-up g-force in flare, and touchdown RODs of $200 \mathrm{fpm}$ and zero-sink (in parenthesis) at ground speed of $250 \mathrm{fps}$ (148 knots) are summarized in Table 1. Again, great sensitivity of the proper flare height on the actual descent 
rate just before the initiation of the flare maneuver is noticeable and partly explains lack of touchdown consistency. A mere 2 feet in flare height or so divide "greaser" from relatively firm landing at $200 \mathrm{fpm}$. Human vision and judgment is normally not that sophisticated to identify 2 - $\mathrm{ft}$ height differences at $50 \mathrm{ft}$ eye heights in a moving and rotating cockpit. Additionally, a mere delay of 300 milliseconds to flare-altitude call-out and initiation of flare plays a significant role in touchdown impact intensity (Daidzic, 2009c).

As the flare height increases using ever gentler pull-up loads so does the sensitivity of the correct flare height on the vertical speed. The uncertainty due to pitch-up flight load is quite large as seen from the total differential:

$$
\Delta h_{F L}=\frac{\partial h_{F L}}{\partial \dot{h}_{A P P}} \delta \dot{h}_{A P P}+\frac{\partial h_{F L}}{\partial \dot{h}_{T D}} \delta \dot{h}_{T D}+\frac{\partial h_{F L}}{\partial\left(\Delta n_{F L}\right)} \delta\left(\Delta n_{F L}\right)
$$

Where partial derivatives defining individual uncertainties are:

$$
\frac{\partial h_{F L}}{\partial \dot{h}_{A P P}}=\frac{R O D_{A P P}}{g \Delta n_{F L}} \quad \frac{\partial h_{F L}}{\partial \dot{h}_{T D}}=\frac{-R O D_{T D}}{g \Delta n_{F L}} \quad \frac{\partial h_{F L}}{\partial\left(\Delta n_{F L}\right)}=\frac{-R O D_{A P P}^{2}}{2 g\left(\Delta n_{F L}\right)^{2}}
$$

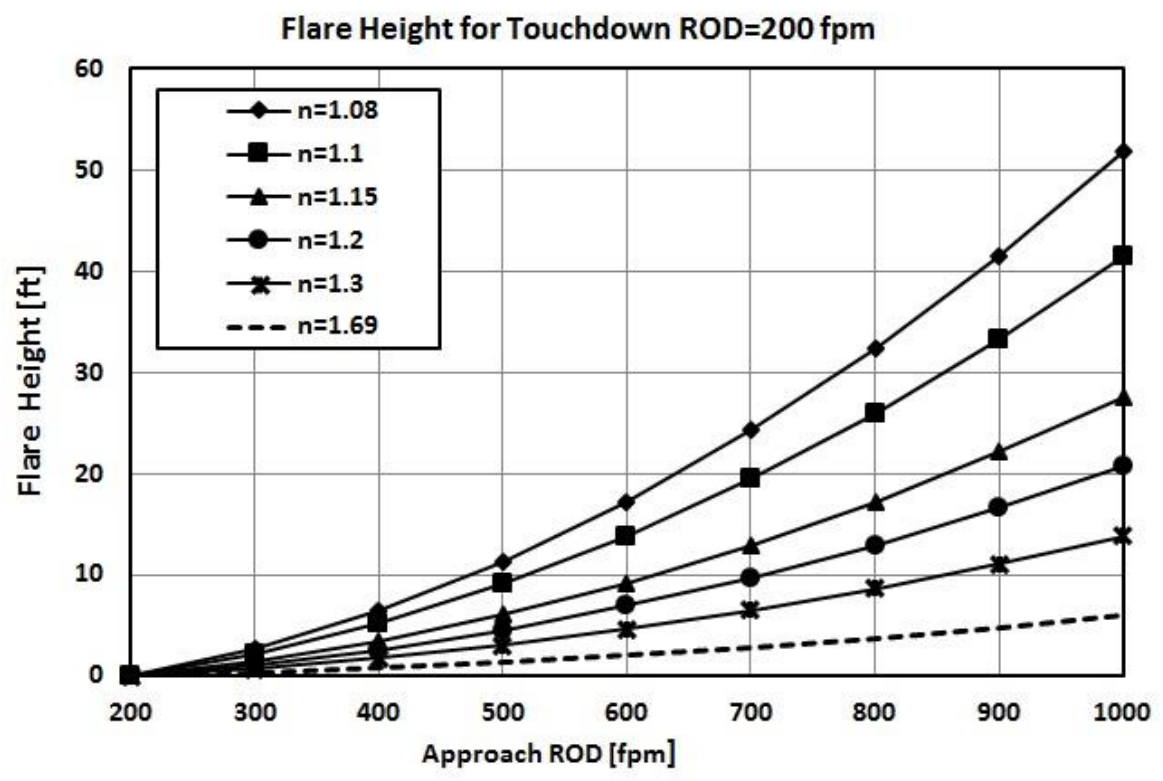

Figure 2. Flare height as a function of approach ROD's and different pull-up gforces at $200 \mathrm{fpm}$ vertical touchdown speeds. 


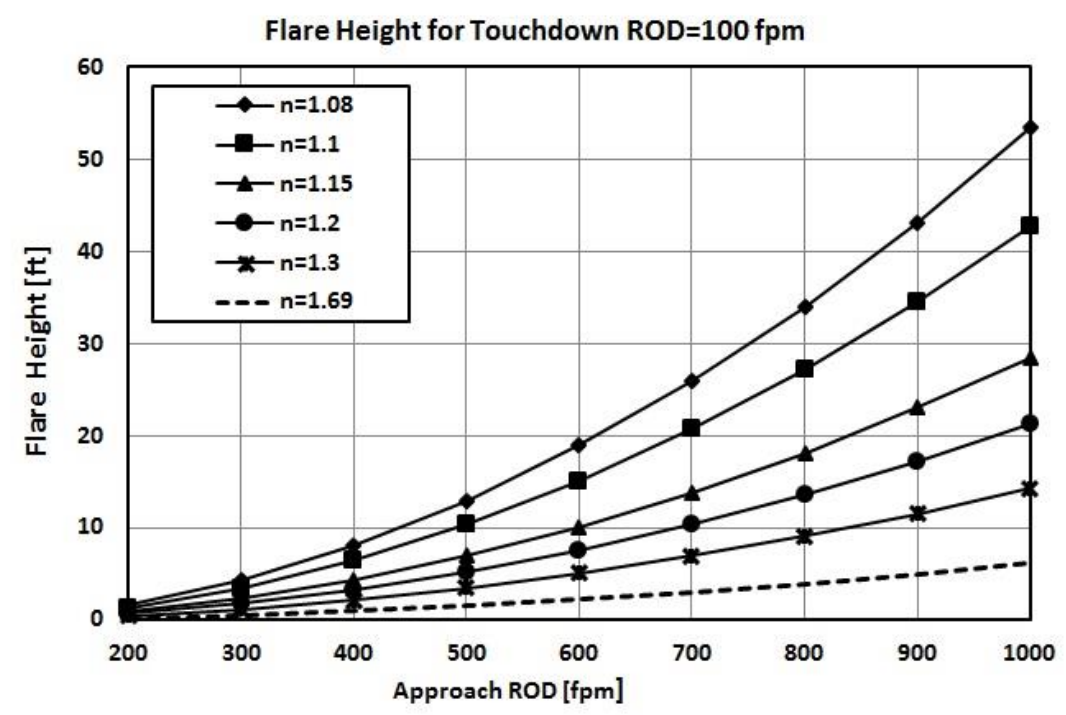

Figure 3. Flare height as a function of approach ROD's and different pull-up gforces at $100 \mathrm{fpm}$ vertical touchdown speeds.

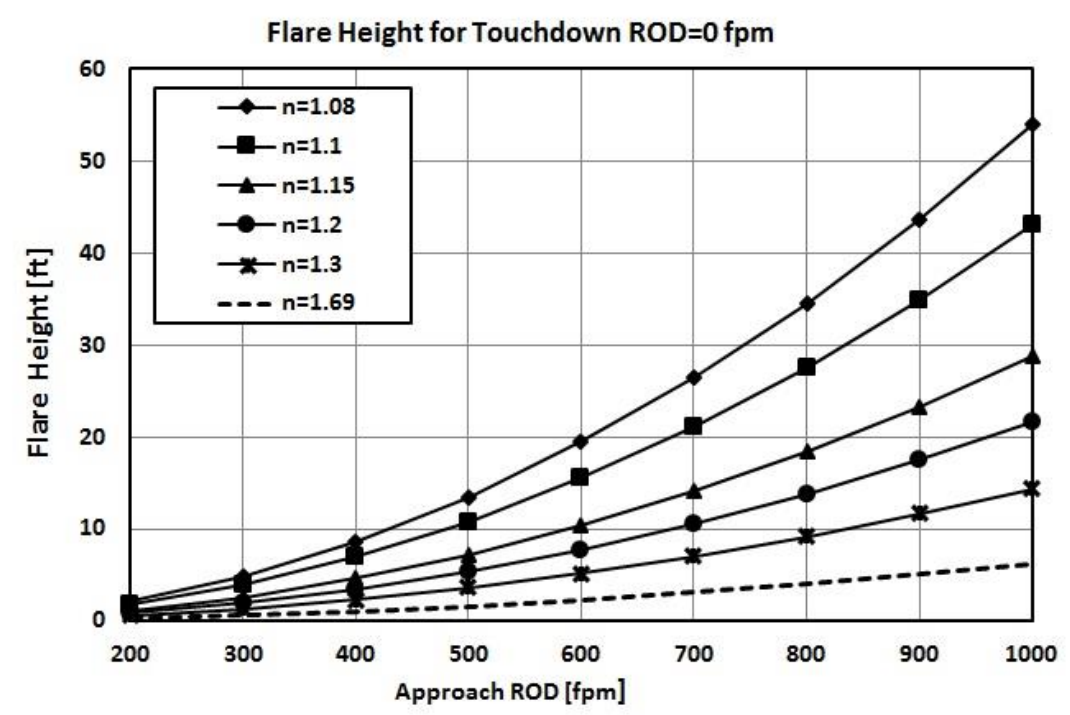

Figure 4. Flare height as a function of approach ROD's and different pull-up gforces at $0 \mathrm{fpm}$ (tangential, zero-sink) vertical touchdown speeds.

As seen in Figures 2, 3 and 4 and particularly from the third partial derivative above (Equation 2), the flare height is very sensitive on the magnitude 
of the pull-up load. The horizontal distance in flare can be calculated from Daidzic \& Shrestha (2008):

$$
L_{F L}=\frac{v_{F L G S}^{2}}{g \cdot \Delta n_{F L}} \sin \left(\gamma_{A P P}-\gamma_{T D}\right) \approx \frac{v_{F L G S}^{2}}{g \cdot \Delta n_{F L}}\left(\gamma_{A P P}-\gamma_{T D}\right)
$$

The total air distance covered from crossing the runway threshold (at $\mathrm{TCH})$ to the touchdown point is:

$$
L_{T D}=\frac{T C H-h_{F L}}{\tan \gamma_{A P P}}+L_{F L}
$$

The minimum distance in flare is actually no-flare $\left(\gamma_{T D}=\gamma_{A P P}\right)$ landing where, $L_{F L \text { min }} \approx h_{F L} / \gamma_{A P P}=0$. This is really hard and damaging on landing gear and occupants and is avoided at all cost. If the flare height is finite positive value, the extra distance covered in flare beyond the point of the straight descent is:

$$
\Delta L_{F L}=L_{F L}-L_{\min }=\frac{h_{F L}}{\gamma_{A P P}} \cdot\left(\frac{\gamma_{A P P}-\gamma_{T D}}{\gamma_{A P P}+\gamma_{T D}}\right)
$$

\begin{tabular}{|c|c|c|}
\hline $\mathbf{R O D}_{\mathrm{APP}}[\mathbf{f p m}]$ & $\begin{array}{c}\text { Gear Height [ft] } \\
\text { ROD } \\
\text { ned }_{\mathrm{FL}}=1.08, \mathrm{q}_{\mathrm{FL}}=0.59 \mathrm{feg} \mathrm{deg} / \mathrm{s}\end{array}$ & $\begin{array}{c}\text { Gear Height [ft] } \\
\text { ROD } \\
\text { n }_{\mathrm{FL}}=1.2, \mathrm{q}_{\mathrm{FL}}=1.48 \mathrm{deg} / \mathrm{s}\end{array}$ \\
\hline 1000 & $51.76(53.92)$ & $20.70(21.57)$ \\
\hline 900 & $41.52(43.67)$ & $16.61(17.47)$ \\
\hline 800 & $32.35(34.51)$ & $12.94(13.80)$ \\
\hline 700 & $24.26(26.42)$ & $9.70(10.57)$ \\
\hline 600 & $17.25(19.41)$ & $6.90(7.76)$ \\
\hline 500 & $11.32(13.48)$ & $4.53(5.39)$ \\
\hline
\end{tabular}

Table 1

Flare height at 250 fps (148 knots).

As a consequence the additional distance covered in curved flare maneuver is a linear function of flare height. Increasing the flare height by $20 \%$ would also require gentler round-out maneuver (lower flare flight load) and increase the flare distance by $20 \%$. The longest flare distance is achieved for 
tangential touchdowns where, $\gamma_{T D}=0$, and assuming the uniform flare flight load. Equation 5 can be rewritten in the following form which also accounts for wind:

$$
\Delta L_{F L}=\frac{R O D_{A P P}^{2}}{2 \cdot g \cdot \gamma_{A P P} \cdot \Delta n_{F L}} \cdot\left(1-\frac{\gamma_{T D}^{2}}{\gamma_{A P P}^{2}}\right)
$$

The horizontal distance covered in flare is proportional to the square of the approach ROD. It is simultaneously inversely proportional to the approach vertical flight path and the constant flare flight load.

For example, an airplane flying a no-wind approach at $250 \mathrm{ft} / \mathrm{s}$ (148 kts) and $3^{\circ}$ glidepath has approach ROD of $13.1 \mathrm{ft} / \mathrm{s}$ or $786 \mathrm{ft} / \mathrm{min}$. The minimum horizontal distance covered from the $30 \mathrm{ft}$ flare height is then $572 \mathrm{ft}$ while maintaining glidepath angle. The "proper" flare height according to Equation 1 would be $33.3 \mathrm{ft}$ at flare load of 0.08 to deliver a constant-radius arc for tangential touchdown.

If the airplane achieves zero-sink touchdown ("greaser") in a smooth curved path, the horizontal distance covered in flare will be 1,270 ft from the 33.3 $\mathrm{ft}$ flare height with flare flight load of $1.080\left(\Delta n_{F L}=0.08\right)$ or constant pull-up load of 0.59 degrees/s. In reality, ground effect and its influences on vortex drag, pitching moment, tail effectiveness, etc., must be taken into account. If the airplane landing gear crossed runway threshold at $50 \mathrm{ft}$ on a $3^{0}$ glidepath, additional $319 \mathrm{ft}$ will be covered in air before starting flare, resulting in the total air distance to touchdown of $1589 \mathrm{ft}$ - assuming perfect conditions.

The horizontal flare distance versus flare flight load at selected $250 \mathrm{fps}$ (148 knots) flare airspeed is presented in Figure 5 for three different touchdown angles using the Equation 3. Obviously, gentle flare starting at higher flare heights would require excessively longer horizontal distances. This analysis does not take into account any ballooning, excessive floating, and bouncing which would add additional distance and possibly result in an overrun.

However, the real flight trajectory during the landing flare looks more like the one presented in Figure 6. The illustration in Figure 1 is just an idealization of the flare maneuver. The initial response to elevator up control input is in airplane accelerating downward, increasing ROD, and losing altitude (or going below the glidepath/glideslope). It also becomes clear why no-flare is sometimes better than late flare. And as it will be shown later, rather than pull-up it is often better to 
push-over in late flare provided pitch angle does not become too flat for touchdown and risking the nose-gear strike first.

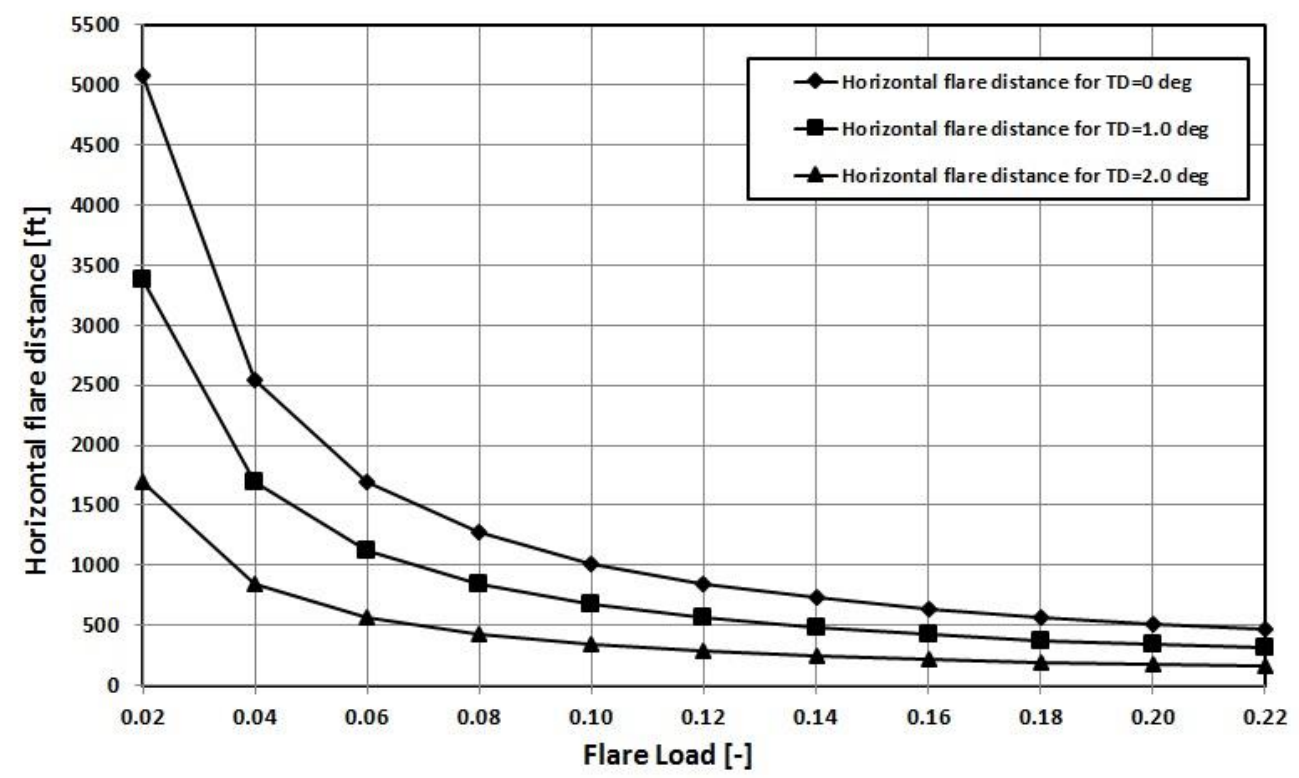

Figure 5. Flare distance as a function of pull-up g-load and touchdown angle at ground speed of 148 knots.

Thus, the main goal of this article is to showcase a relatively simple, yet quite powerful and realistic (on short time-scales) adverse effect of elevator control (AEE) in conventional airplane designs. Many computations for a selected transport-category aircraft will be presented for specific control inputs. A new landing technique is proposed which will utilize AEE to the pilot's benefit making touchdowns more accurate and reducing the required runway lengths.

\section{Mathematical model of Adverse Elevator Effect}

The mathematical model for the simultaneous pitching and plunge (heave) motion presented here is based on the linearized short-term (1 to 2 seconds) longitudinal dynamics of airplane in landing flare. Simple, yet powerful mathematical models have been developed in time and complex domain with Laplace/Heaviside transfer functions (TF) defining responses to arbitrary elevator control inputs. Only small perturbations of rigid-body aircraft about the equilibrium (reference) flight conditions are considered resulting in a set of two to three ordinary differential equations (ODE) describing simple longitudinal dynamics with up to three degrees-of-freedom (3-DOF). The longitudinal 
dynamics is decoupled from the lateral-directional dynamics and the small difference in the lateral moment of inertia between the principal body axis and the stability axis has been neglected (Roskam, 2007). The AEE is investigated here on the time-scales which are somewhat shorter than the oscillatory and heavily damped short-period (SP) oscillatory pitching dynamics (Etkin, 1959, 2005; Kolk, 1961; Seckel, 1964; Nelson, 1998; Phillips, 2004, Roskam, 2007; Schmidt, 2012; Stengl, 2004; Stevens \& Lewis, 2003). Of many stability derivatives only the elevator control effect is considered along the perturbed lift force on the main wing. Pitch stiffness and pitch damping are neglected. Ground effect has not been simulated for simplicity.

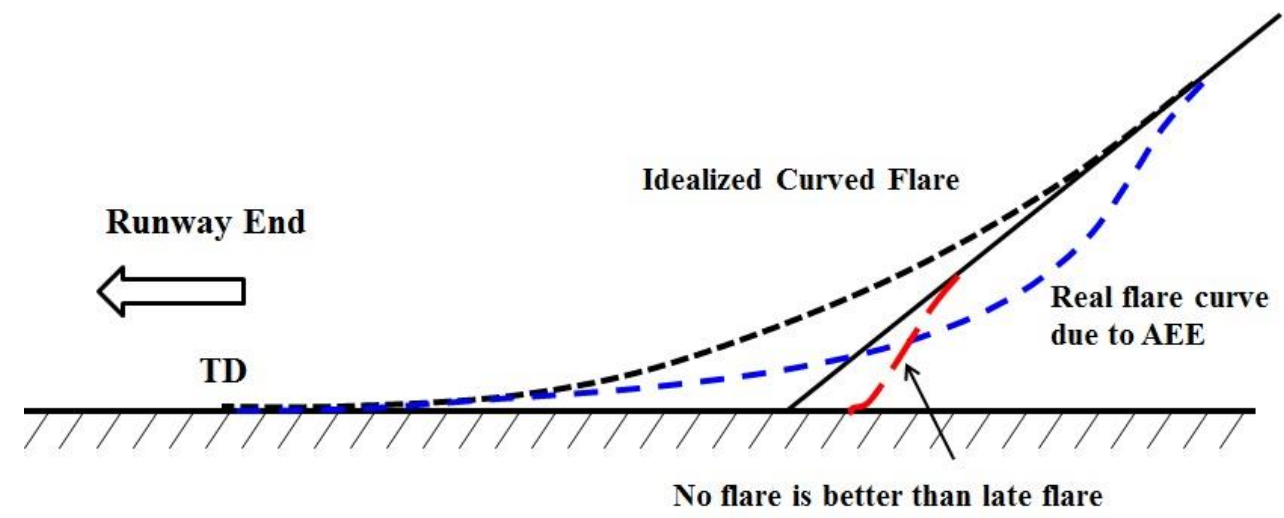

Figure 6. Illustration of idealized and real flare dynamics with AEE. Not so scale. Angles and distances are highly exaggerated for better visual representation.

A transport-category airplane in stabilized landing approach is typically descending at 10-15 feet/seconds (600-900 fpm). Usually, such airplane would start flare at gear heights of 20-40 feet. Larger "jumbos" and "super-jumbos" could start pre-flare maneuver at even higher heights. Some large airplanes with high aspect-ratio and wingspan have such a massive ground-effect in flare that very little elevator control is required (Davis, 1971; Denton, 1993). If the pilot does not flare the aircraft, and depending on the approach ROD, it would take only about 3 seconds before the landing gear impacts runway surface at unacceptably high vertical speeds.

It is assumed that a landing airplane entered the flare height at a steady vertical flight path angle, steady ROD, and constant approach AOA. The variables of interest can be written as a sum of steady-state (reference or equilibrium) and small perturbation values: 
$\alpha(t)=\bar{\alpha}_{A P P}+\Delta \alpha(t) \quad \theta(t)=\bar{\theta}_{A P P}+\Delta \theta(t) \quad \gamma(t)=\bar{\gamma}_{A P P}+\Delta \gamma(t) \quad h(t)=\bar{h}_{A P P}+\Delta h(t)$

The initial conditions (ICs) for perturbed states are by definition all zero. Airspeed change during landing flare in ground effect is small and is neglected. The kinematic condition between AOA, pitch angle, and vertical flight path (glidepath) angle yields, $\theta=\alpha+\gamma-\alpha_{i}$. Since the incidence angle $\left(\alpha_{i}\right)$ is fixed any pitch change consists of the AOA and flight path-angle change $(\Delta \theta=\Delta \alpha+\Delta \gamma)$. A pure-pitching motion exists when $\mathrm{CG}$ is constrained and all the pitch change goes into AOA change alone with no flight-path variation.

Small-perturbation flight dynamics equations (Etkin, 1959, 2005; Kolk, 1961; Seckel, 1964; Abzug \& Larrabee, 1997; Nelson, 1998; Stevens \& Lewis, 2003; Phillips, 2004, Stengel, 2004; Roskam, 2007; Schmidt, 2012) are originally due to Bryan in 1911 and have been an invaluable tool in development of new aircraft and flight simulation ever since. All partial derivatives in linearly truncated Taylor expansion are evaluated at the reference (equilibrium) conditions (steady-state flight). The only control force and moment discussed here are those coming from an elevator. Several simplifying assumptions are made in describing the AEE of a rigid-body conventional-tail aircraft. A change of airspeed is negligible for short time periods and thus forward speed-damping terms have been neglected $\left(X_{u}, Z_{u}, M_{u}\right)$ in the absence of any thrust perturbation. The entire force equation in the $\mathrm{X}$-direction (longitudinal) can thus be dropped. Pitch damping terms $\left(C_{m_{q}}, C_{m_{\dot{\alpha}}}\right)$, pitch stiffness $\left(C_{m_{\alpha}}\right)$, and vertical damping $\left(C_{z_{q}}\right)$ have been also neglected. A 3-DOF linear time-invariant (LTI) model for quasi-steady motion in vertical plane is only considered.

\section{Aircraft response to an arbitrary elevator input and pure-pitching restriction}

A simple solution of short-time flare dynamics can be obtained from the "pure-pitching" kinematic approximation (Nelson, 1998) resulting in all pitch change going into increase in AOA only, while the flight path angle stays constant $(\Delta \gamma=0)$. In such case, the simple linearized mathematical model becomes:

$$
\begin{aligned}
& \Delta \ddot{h}(t)=Z_{\alpha} \Delta \alpha(t)+Z_{\delta_{e}} \Delta \delta_{e}(t) \\
& \Delta \ddot{\theta}(t)=M_{\delta_{e}} \Delta \delta_{e}(t) \\
& \Delta \alpha(t)=\Delta \theta(t) \Rightarrow \Delta \gamma(t)=0 \\
& \Delta \dot{\theta}(t)=\Delta p(t)
\end{aligned}
$$


The aircraft stability-axes (Roskam, 2007; Stevens \& Lewis, 2003) are used with the body z-axis pointing downward while the vertical component of the earth-fixed inertial reference system is pointing upward. The first ODE is simply a balance of perturbed forces in the vertical direction ("heave" motion). The second ODE represents the conservation of pitching angular momentum. The third equation describes the instantaneous kinematic condition for the purepitching dynamics. The forces and moments (stability derivatives) in Equation 7 are expressed as:

$$
\begin{aligned}
& Z_{\alpha}=\frac{1}{m}\left(\frac{\partial Z}{\partial \alpha}\right)_{0}=C_{Z_{\alpha}} \frac{Q S}{m}=-C_{L_{\alpha}} \frac{Q S}{m} \quad\left[\mathrm{ft} / \mathrm{s}^{2}\right] \\
& Z_{\delta_{e}}=\frac{1}{m}\left(\frac{\partial Z}{\partial \delta_{e}}\right)_{0}=C_{Z_{\delta_{e}}} \frac{Q S}{m}=-C_{L_{\delta_{e}}} \frac{Q S}{m}\left[\mathrm{ft} / \mathrm{s}^{2}\right] \\
& M_{\delta_{e}}=\frac{1}{I_{y y}}\left(\frac{\partial M}{\partial \delta_{e}}\right)_{0}=C_{m_{\delta_{e}}} \frac{Q S \bar{c}_{w}}{I_{y y}}\left[1 / \mathrm{s}^{2}\right]
\end{aligned}
$$

The longitudinal stability and control coefficients can be or measured in flight tests or wind tunnels or modeled using standard methods (Abzug \& Larrabee, 1997; Kolk, 1961; Seckel, 1964; Russell, 1996; Nelson, 1998; Phillips, 2004; Roskam, 2007; Schmidt, 2012; Stengl, 2004; Stevens \& Lewis, 2003).

Aircraft's vertical body-axis follows the right hand rule and points downward while the Earth-fixed vertical axis points upward and so the sign will change. Perhaps it is more intuitive to think about the tail lift perturbation as expressed in more familiar lift increment form:

$$
\begin{aligned}
& \Delta L_{t}=\Delta L_{\delta_{e}}=C_{L_{\delta_{e}}} \Delta \delta_{e} Q S=-m Z_{\delta_{e}} \Delta \delta_{e} \\
& \Delta M_{t}=\Delta M_{\delta_{e}}=-\Delta L_{t} \cdot l_{t}=-I_{y y} M_{\delta_{e}} \Delta \delta_{e}
\end{aligned}
$$

The elevator movement (down or up) will define the sign of the tail lift and the pitching moment change. By aircraft stability and control theory convention upward elevator (pitching up) is taken as negative (Nelson, 1998; Phillips, 2004; Roskam, 2007). An elevator temporal movement can be defined by any control input and can be a Dirac's impulse, Heaviside's step, ramp, parabolic, sinusoidal, finite pulse, doublet, or anything desired and tailored.

The final set of longitudinal dynamic equations for pure-pitching flight perturbation becomes: 


$$
\begin{aligned}
& \Delta \ddot{\theta}(t)=-R \cdot \Delta \delta_{e}(t) \\
& \Delta \ddot{\alpha}(t)=\Delta \ddot{\theta}(t) \\
& \Delta \ddot{h}(t)=K \cdot \Delta \alpha(t)+P \cdot \Delta \delta_{e}(t) \\
& u(t)=\Delta \delta_{e}(t)
\end{aligned}
$$

The function $u(t)$ is control input (elevator deflection). The direction of elevator deflection will define the sign (positive or negative) so that both elevatorup $\left(\Delta \delta_{e}<0\right)$ and elevator-down $\left(\Delta \delta_{e}>0\right)$ deflections can be simulated. Up elevator has negative sign but induces positive pitching moment and rate (increasing pitch angle). The various constants in Equation 9 are defined as:

$$
\begin{aligned}
& m=\frac{W}{g} \quad C_{L_{\alpha}}=\frac{\partial C_{L}}{\partial \alpha} \quad Q=\frac{1}{2} \rho v_{0}^{2} \\
& K=\frac{Q S C_{L_{\alpha}}}{m} \quad P=\frac{\Delta L_{t}}{m} \quad R=\frac{\Delta L_{t} \cdot l_{t}}{I_{y y}} \quad b=\frac{K}{v_{0}}
\end{aligned}
$$

The parameter $K$ can be described as the acceleration due to main wing lift increment, parameter $P$ is the vertical acceleration due to tail elevator lift change, and $R$ is the pitching angular acceleration. Important parameter in main wing lift production is the product, $K \times R$. For many transport-category airplanes this product of vertical and angular (pitching) accelerations is almost constant. Pinsker (1969) arrived to similar set of equations as we did.

\section{Aircraft response to an arbitrary elevator input and free flight path}

In this analysis the restriction of pure-pitching motion will be removed and the vertical flight-path angle will become independent (free flight). The conservation of angular momentum along airplane's lateral axis (pitch rotation) for arbitrary elevator input becomes:

$$
I_{y y} \frac{d^{2}(\Delta \theta)}{d t^{2}}=I_{y y} \Delta \ddot{\theta}(t)=\Delta M_{t}=-\Delta L_{t} \cdot l_{t} \cdot u(t)
$$

The balance of vertical forces will include the increase in the elevator downward force and the time-dependent increase in main-wing lift: 


$$
\frac{W}{g} \frac{d^{2}(\Delta h)}{d t^{2}}=\frac{W}{g} \Delta \ddot{h}(t)=C_{L_{\alpha}} \Delta \alpha(t) \cdot Q \cdot S+\Delta L_{t} \cdot u(t)
$$

The third ODE describing vertical flight path (glidepath) change is obtained from the kinematic condition and constant-airspeed approximation:

$$
\sin \gamma \approx \gamma=\frac{\dot{h}}{v_{0}} \Rightarrow \Delta \gamma=\frac{\Delta \dot{h}}{v_{0}} \quad \text { and } \quad \Delta \dot{\gamma}=\frac{\Delta \ddot{h}}{v_{0}} \approx \frac{g}{v_{0}} \Delta n_{F L}
$$

The kinematic condition in free flight is very different from the one in pure-pitching mode:

$$
\Delta q=\Delta \dot{\theta}=\Delta \dot{\alpha}+\Delta \dot{\gamma} \quad \text { and } \quad \Delta \dot{\theta}=\Delta \dot{\alpha}+\frac{\Delta \ddot{h}}{v_{0}} \Rightarrow \Delta \ddot{\theta}=\Delta \ddot{\alpha}+\frac{\Delta \dddot{h}}{v_{0}}
$$

The final set of ODEs describing the short-term AEE longitudinal flight dynamics in free-flight at small flight angle is:

$$
\Delta \ddot{\theta}(t)=-R \cdot u(t) \quad \Delta \dot{\alpha}=\Delta \dot{\theta}-\frac{\Delta \ddot{h}}{v_{0}} \quad \Delta \ddot{h}(t)=K \cdot \Delta \alpha(t)+P \cdot u(t)
$$

The free-flight longitudinal dynamics model is more complex than for pure-pitching approximation as it allows for the change of the AOA and flight path angles independently.

\section{Methods and Materials}

Aircraft-response based on the particular pilot-controlled elevator input are investigated. Impulse and step control inputs were used to investigate aircraft longitudinal dynamics and AEE. Although these simple control inputs are just an approximation of how pilots normally would command pitch changes, they nevertheless unravel the mystery behind AEE. Other delays in control system are neglected.

The system of coupled linear ODEs describing rigid-airplane longitudinal dynamics can be conveniently solved using the powerful space-state analysis (Nelson, 1998; Phillips, 2004; Stengl, 2004; Schmidt, 2012). However, for the simple single-input system (elevator deflection), the Laplace transforms (Spiegel, 1965) are chosen. The Laplace transformation is also a very powerful 
mathematical technique with a beautiful property that it converts ODE into algebraic functions in Laplace complex domain. Without describing details of the Laplace transform properties, the transform-pairs used here are defined as:

$$
\begin{array}{llll}
T(s)=L[\Delta \theta(t)], & \Delta \theta(t)=L^{-1}[T(s)] ; & A(s)=L[\Delta \alpha(t)], & \Delta \alpha(t)=L^{-1}[A(s)] \\
H(s)=L[\Delta h(t)], & \Delta h(t)=L^{-1}[H(s)] ; & U(s)=L[u(t)], & u(t)=L^{-1}[U(s)]
\end{array}
$$

An interested reader is welcome to consult many great mathematical books written on integral transforms and Laplace transform in particular. A good, simple, and very popular introductory text used here is by Spiegel (1965).

\section{Solution to pure-pitching longitudinal flight dynamics approximation}

In Laplace domain the system of ODE (Equation 9) becomes a system of three algebraic equations with three unknowns. If the elevator is up then the deflection is negative and the incremental tail-lift is directed downward generating a positive pitching motion (upward). The Laplace transforms of system given by Equation 9 becomes:

$$
s^{2} T(s)=-R \cdot U(s) \quad A(s)=T(s) \quad s^{2} H(s)=K \cdot A(s)+P \cdot U(s)
$$

and:

$T(s)=-R \frac{U(s)}{s^{2}} \quad A(s)=T(s) \quad H(s)=K \cdot \frac{T(s)}{s^{2}}+P \frac{U(s)}{s^{2}}$

The TFs for elevator control input can be defined for pitch, AOA, and altitude responses:

$$
\frac{T(s)}{U(s)}=-\frac{R}{s^{2}} \quad \frac{A(s)}{U(s)}=\frac{T(s)}{U(s)}=-\frac{R}{s^{2}} \quad \frac{H(s)}{U(s)}=-\frac{K \cdot R}{s^{4}}+\frac{P}{s^{2}}
$$

For an impulse input, the Laplace transform of the elevator control function is simply $U(s)=-1$ (elevator up) or $U(s)=+1$ (elevator down). Performing the inverse Laplace transform of Equation 15 for the impulse elevator up deflection (approximating quick jerk back and release) results in time-domain:

$$
\Delta \theta(t)=\Delta \alpha(t)=R t \quad \Delta h(t)=\frac{K R}{6} t^{3}-P t
$$


For an impulse elevator down control input $U(s)=+1$ (approximating quick jerk forward and release), the temporal solution for pure-pitching altitude and pitch (also AOA) change is:

$$
\Delta \theta(t)=\Delta \alpha(t)=-R t \quad \Delta h(t)=-\frac{K R}{6} t^{3}+P t
$$

If the elevator control input is unit step up (elevator suddenly up and hold that deflection) or, $U(s)=-1 / s$, the temporal height and pitch changes are:

$$
\Delta \theta(t)=\Delta \alpha(t)=R \frac{t^{2}}{2} \quad \Delta h(t)=\frac{K R}{24} t^{4}-P \frac{t^{2}}{2}
$$

For unit step down or, $U(s)=1 / s$, the temporal height and pitch changes in pure-pitching mode become:

$$
\Delta \theta(t)=\Delta \alpha(t)=-R \frac{t^{2}}{2} \quad \Delta h(t)=-\frac{K R}{24} t^{4}+P \frac{t^{2}}{2}
$$

Since all the above functions are analytic, the derivatives with respect to time can be easily found. The characteristic duration (time-constant) of the AEE or inverse altitude response to step control input during which an airplane essentially only rotates around lateral axis is:

$$
\tau_{\text {step }}=\sqrt{\frac{P}{K \cdot R}}=\sqrt{\frac{I_{y y}}{Q \cdot S \cdot C_{L_{\alpha}} \cdot l_{t}}}
$$

For a B747-200 airplane this time constant is about 365 milliseconds (ms) at 148 knots approach speed and SL density altitude. All three important time scales defining the duration of loss of altitude (reverse altitude response), increased (adverse) ROD, and downward acceleration from the moment step elevator input are (Pinsker, 1969):

$$
t_{\Delta h=0} \approx 3.46 \cdot \tau_{\text {step }}, \quad t_{\Delta \dot{h}=0}=\frac{t_{\Delta h=0}}{\sqrt{3}} \approx 2 \cdot \tau_{\text {step }}, \quad t_{\Delta \ddot{h}=0}=\frac{t_{\Delta h=0}}{\sqrt{6}}=\frac{t_{\Delta \dot{h}=0}}{\sqrt{2}} \approx 1.41 \cdot \tau_{\text {step }}
$$


Interestingly, the characteristic step-response time $\tau_{\text {step }}$ does not depend on the magnitude of the elevator force or deflection (up or down), but only on the specific aerodynamic parameters. In the case of impulse input the respective time constant is about $71 \%$ of the step time constant for AEE.

\section{Solution of free-flight longitudinal dynamics}

Applying the Laplace transform to all three ODE (Equation 12) results in the system of three algebraic equations for three unknown functions:

$$
s^{2} T(s)=-R \cdot U(s) \quad s A(s)=s T(s)-\frac{s^{2} H(s)}{v_{0}} \quad s^{2} H(s)=K \cdot A(s)+P \cdot U(s)
$$

The functions for pitch, height and AOA in Laplace domain for arbitrary control input and free-flight condition become:

$$
\begin{aligned}
& T(s)=-R \frac{U(s)}{s^{2}} \quad A(s)=T(s)-\frac{s H(s)}{v_{0}} \\
& H(s)=-K \cdot R \frac{U(s)}{s^{3}(s+b)}+P \frac{U(s)}{s(s+b)}
\end{aligned}
$$

The transfer functions for pitch, AOA, and altitude/height response can be now defined as:

$$
\frac{T(s)}{U(s)}=-\frac{R}{s^{2}} \quad \frac{A(s)}{U(s)}=\frac{T(s)}{U(s)}-\frac{s}{v_{0}} \frac{H(s)}{U(s)} \quad \frac{H(s)}{U(s)}=-\frac{K \cdot R}{s^{3}(s+b)}+\frac{P}{s(s+b)}
$$

Only impulse and step responses for elevator-up control input will be examined. Elevator down is just a mirror image of elevator up control input. For an elevator-up impulse response, where, $U(s)=-1$, the solution to Laplace inversions yields:

$$
\begin{aligned}
& \Delta \theta(t)=R t \\
& \Delta \alpha(t)=\frac{R}{b}\left(1-e^{-b t}\right)+\frac{P}{v_{0}} e^{-b t} \\
& \Delta h(t)=\frac{K R}{2 b}\left(t^{2}-\frac{2 t}{b}\right)+\frac{K R}{b^{3}}\left(1-e^{-b t}\right)-\frac{P}{b}\left(1-e^{-b t}\right)
\end{aligned}
$$


The above analytic solutions (Equation 23) yield values at zero-time:

$\Delta h(0)=0 \quad \Delta \dot{h}(0)=-P \quad \Delta \ddot{h}(0)=P \cdot b$

For an elevator-up step response, where $U(s)=-1 / s$, the solutions for altitude, pitch and AOA, after some quite tedious Laplace inversions and algebraic reductions, yields:

$$
\begin{aligned}
& \Delta \theta(t)=R \frac{t^{2}}{2} \\
& \Delta \alpha(t)=\frac{R t}{b}-\left(1-e^{-b t}\right) \cdot\left(\frac{R}{b^{2}}-\frac{P}{K}\right) \\
& \Delta h(t)=\frac{K R}{6 b}\left(t^{3}-\frac{3 t^{2}}{b}+\frac{6 t}{b^{2}}\right)-\frac{K R}{b^{4}}\left(1-e^{-b t}\right)-\frac{P t}{b}+\frac{P}{b^{2}}\left(1-e^{-b t}\right)
\end{aligned}
$$

These analytic solutions (Equation 24) yield values at initial- or zero-time:

$$
\Delta h(0)=0 \quad \Delta \dot{h}(0)=0 \quad \Delta \ddot{h}(0)=-P
$$

In order to find various inverse-transforms, many Laplace transform properties have been used including the convolution integral, transform of the derivative function, etc. (Spiegel, 1965). Again, all functions are analytic and continuous in the entire time domain and the temporal derivatives can be easily found and are thus not explicitly given here. The free-flight kinematic condition (Equation 11) is satisfied by both (impulse and step) solutions given with Equations 23 and 24.

\section{Results and Discussion}

The responses for an impulse and a step elevator up and down control inputs of various magnitudes in pure-pitching and free-flight are presented. The $\mathrm{AAE}$ and the reverse altitude response is clearly present for the initial short-period after the flight control application as will be shown below.

The evaluation of height, vertical speed, and vertical acceleration for two different impulse elevator up and down inputs is examined first. The acceleration in pitch is constant since pitch stiffness and damping were neglected for short time scales. A geometrical and aerodynamic characteristics of B747-200 have been used as that data is readily available (Nelson, 1998; Philipps, 2004) although 
no great accuracy is claimed. The landing weight used is 550,000 $\mathrm{lb}$, wing reference area is $5,500 \mathrm{ft}^{2}$ with the wingspan of 195 feet, the pitching moment of inertia is 30,000,000 slug- $\mathrm{ft}^{2}$ and the horizontal-tail-to-CG arm is $100 \mathrm{ft}$. The reference/flare speed was taken as 148 knots (250 fps) at SL density altitude.

Tail lift perturbations due to elevator (up or down) applications have been arbitrarily taken as 50,000 and 100,000 lb respectively. In terms of elevator deflection angle where elevator is assumed to be symmetric airfoil with maximum up and down deflections of $45^{\circ}$ in slow speed regime, one obtains:

$$
\Delta \delta_{e}=-\frac{\Delta L_{t}}{C_{Z_{\delta_{e}}} Q S} \quad \Delta \delta_{e}=\frac{\Delta M_{t}}{C_{m_{\delta_{e}}} Q S \bar{c}}=-\frac{\Delta L_{t} \cdot l_{t}}{C_{m_{\delta_{e}}} Q S \bar{c}} \quad C_{m_{\delta_{e}}}=C_{Z_{\delta_{e}}} \frac{l_{t}}{\bar{c}}
$$

If the elevator power is calculated for B747-200 to be $C_{Z_{\delta_{e}}}=-0.4$ and $C_{m_{\delta_{e}}}=-1.43$ at a given approach airspeed and SL ISA conditions, the 50,000 lb tail vertical lift increment corresponds to elevator (up or down) deflection of $17.5^{0}$. For $100,000 \mathrm{lb}$ tail-lift increment, a $35^{0}$ elevator deflection is correspondingly required. These forces and deflections are, in fact, excessive for normal landings, but serve to show that AEE's pitch time-lag does not depend on the magnitude of tail lift perturbation.

The first result of computations will be height, vertical speed and acceleration for impulse upward elevator movement for pure-pitching motion and 50,000 and 100,000 lb tail-lift increments as shown in Figure 7. Similar results are shown in Figure 8, but this time the elevator deflection is downward creating initially an upward motion, a sort of, proverse elevator effect. The initial upward motion is just a mirror image of downward motion (AEE) and will thus not be repeated for every case. As expected, the time delay (lag) due to AEE is independent of the tail lift magnitude. The results for the pure-pitching flight dynamics and elevator step up and down control deflections are shown in Figures 9 and 10 respectively. Again, the AEE is clearly noticeable and the time-lag is actually longer than for the case of impulse control input (about $1.25 \mathrm{~s}$ versus 0.9 $\mathrm{s}$ for the impulse control input). Elevator-down control input is just a mirrorimage of elevator-up response.

Analog results for free-flight longitudinal dynamics are shown in Figures 11 and 12 for impulse and step elevator-up response. Again, the same 50,000 and $100,000 \mathrm{lb}$ tail lift increments were used. Since the effect of elevator down is just a mirror image of the elevator-up response that was presented earlier it has not been simulated separately. A response in pitch, AOA and height for an impulse 
elevator up control input is shown in Figure 11. A step response is shown in Figure 12. Again, the AEE is quite obvious introducing longer delay in step response than for impulse control input. The magnitude of the time-lag is somewhat moderated compared to pure-pitching motion, but not much, suggesting that the initial response to elevator control input is a linear combination of downward acceleration and pure rotation around the lateral axis.
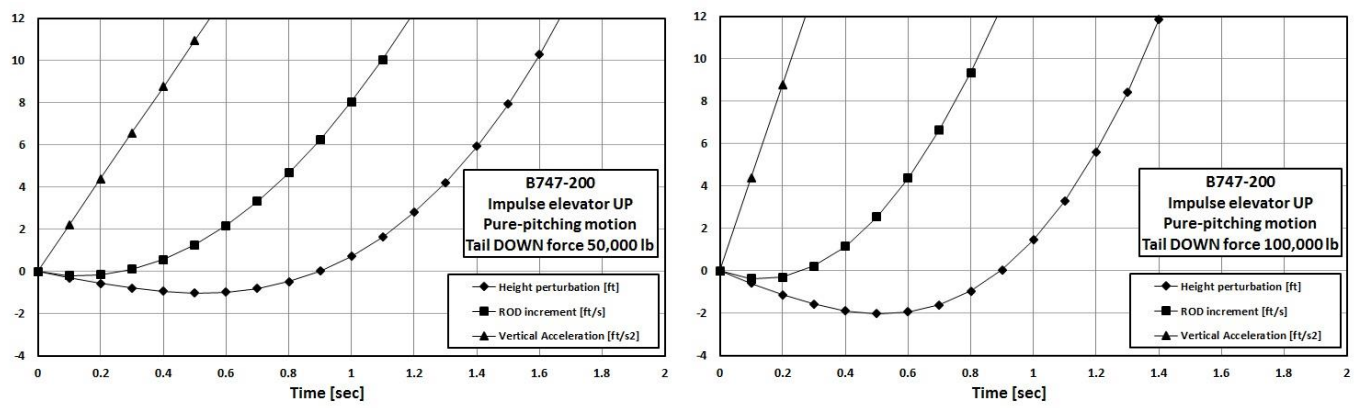

Figure 7. Height, vertical speed, and vertical acceleration perturbations due to impulse elevator-up 50,000 (LHS) and 100,000 lb (RHS) tail lift change input for pure-pitching restricted flight dynamics.
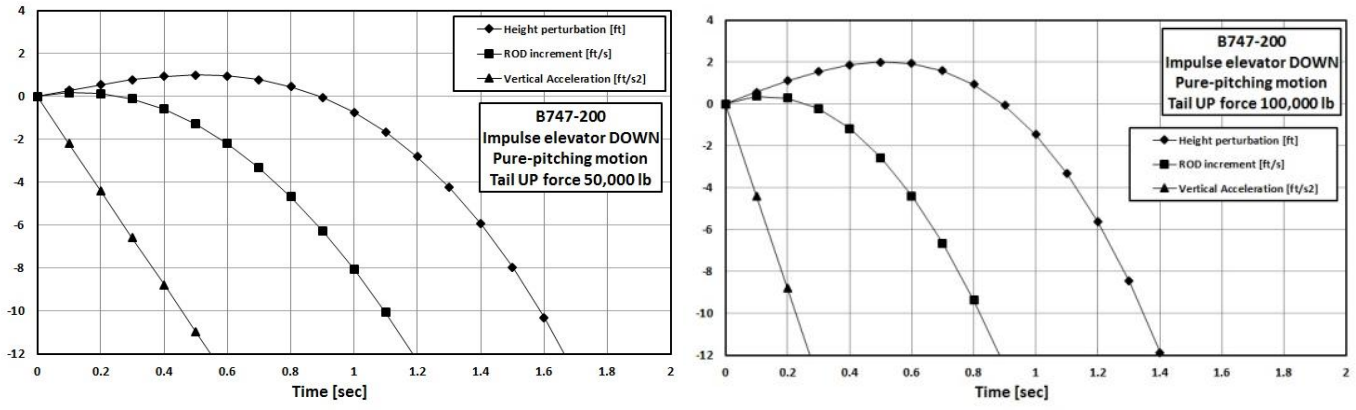

Figure 8. Height, vertical speed, and vertical acceleration perturbations due to impulse elevator-down 50,000 (LHS) and 100,000 lb (RHS) tail lift change input for pure-pitching restricted flight dynamics.

Another useful observation is that short impulses (jerks) on elevator introduce less adverse effects than step input (pull and hold). As will be shown in subsequent articles, ramp elevator input (progressively increasing elevator up) introduces even larger AEE lag times. This certainly has to do with the amount of energy introduced by the elevator deflection. 

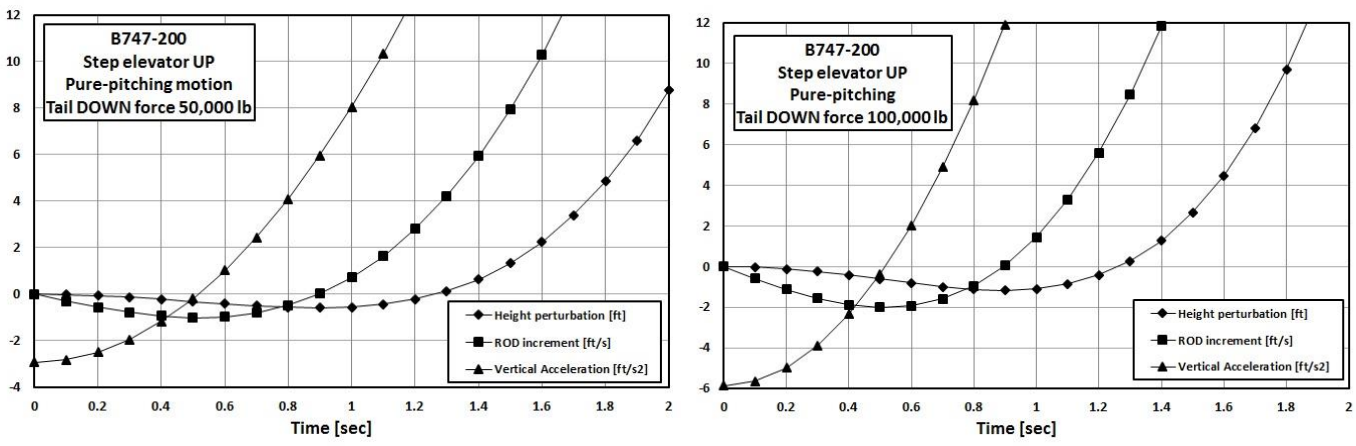

Figure 9. Height, vertical speed, and vertical acceleration perturbations due to step elevator-up 50,000 (LHS) and 100,000 lb (RHS) tail lift change input for pure-pitching restricted flight dynamics.
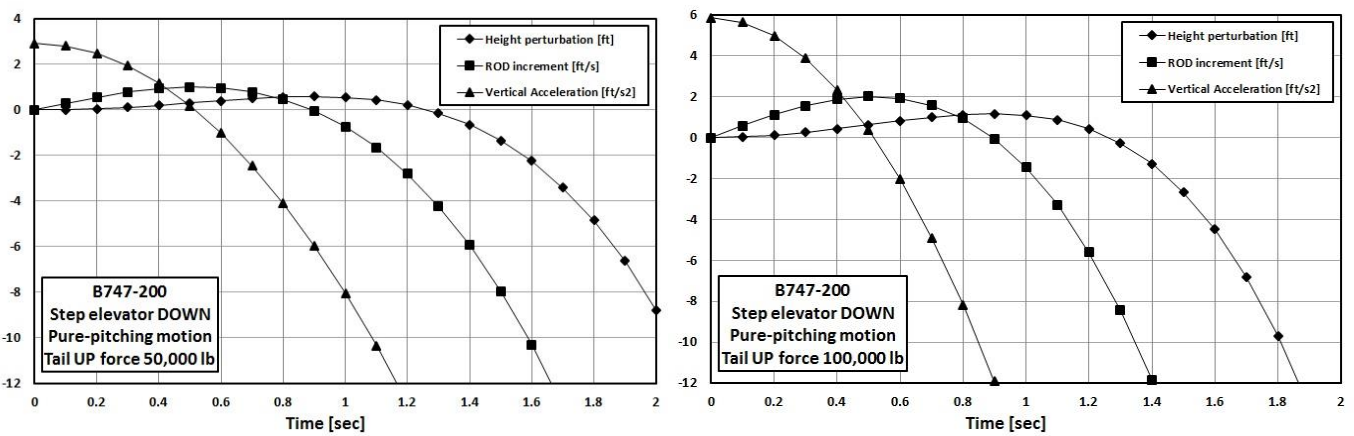

Figure 10. Height, vertical speed, and vertical acceleration perturbations due to step elevator-down 50,000 (LHS) and 100,000 lb (RHS) tail lift change input for pure-pitching restricted flight dynamics.
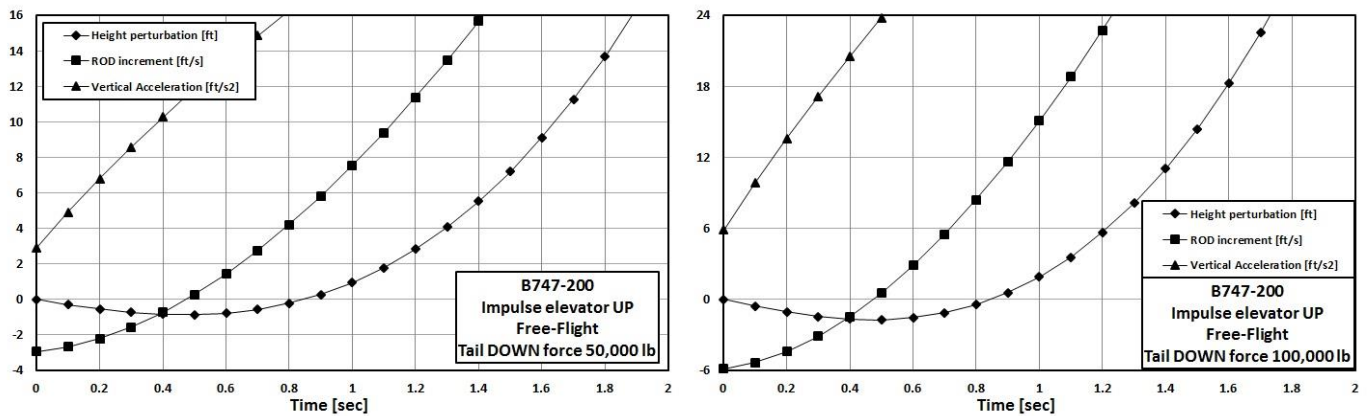

Figure 11. Height, vertical speed, and vertical acceleration perturbations due to impulse elevator up 50,000 (LHS) and 100,000 lb (RHS) tail lift change input for free-flight dynamics. 
The intensity of the tail-down (or up) lift does not affect the lag-time constant for a particular control input (impulse or step). Only the magnitude of adverse effects, such as reverse, altitude response, vertical acceleration and speed changes are affected. In a way that is to be expected from a linear isochronous model.
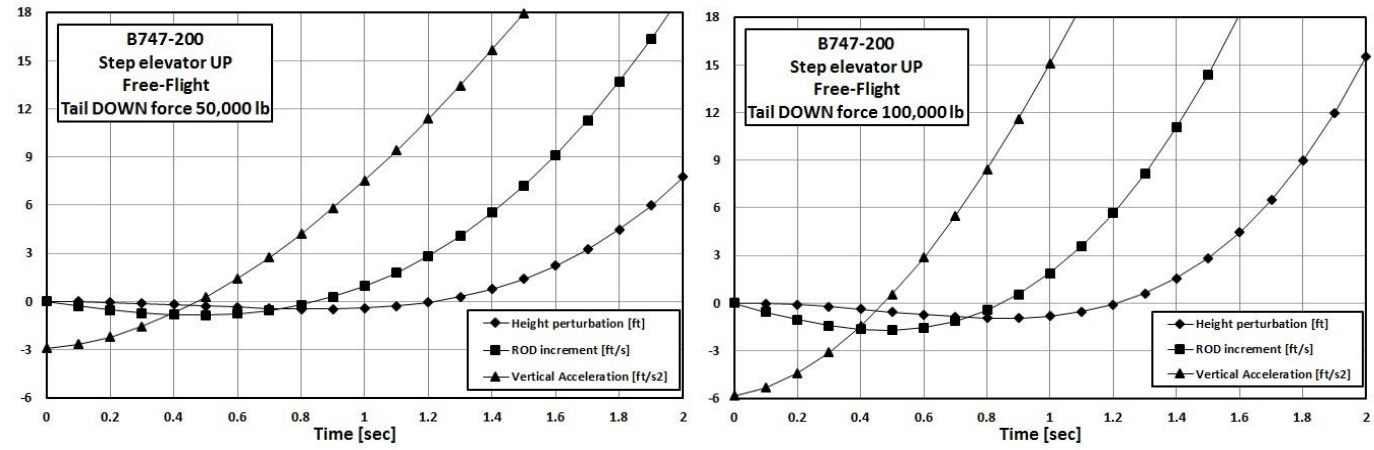

Figure 12. Height, vertical speed, and vertical acceleration perturbations due to step elevator up 50,000 (LHS) and 100,000 lb (RHS) tail lift change input for free-flight dynamics.

The results of the unrestricted longitudinal small-perturbations flight dynamics full simulations for a $10^{0}$ step elevator-up control input for B747-200 utilizing in-house developed flight simulation dynamics programs in MATLAB ${ }^{\mathrm{TM}}$ and optimizing Fortran 95/2003 compiler with IMSL subroutines are shown in Figure 13. Advanced Runge-Kutta variable-step methods for numerical solution of system of ODEs were utilized. The full model includes all stiffness and damping terms and fully models longitudinal dynamics and control effects in freeflight. This simulation result is used only to validate the assumptions made in the simple longitudinal flight models utilized here (Equations 9 and 21). No attempt is made here to describe details of the full longitudinal dynamics model due to size and complexity restrictions. From the state-space analysis of the full model the times $T_{1 / 2}$ to halve the short-period (SP) and the long-period (phugoid or LP) modes for B747-200 are 1.078 and 84.35 seconds respectively. SP and LP damping constants are 0.939 and 0.0588 respectively. The fundamental decaying oscillatory periods signifying dynamic stability were 9.167 and $44.97 \mathrm{~s}$ respectively. Accordingly, the AEE (reverse altitude effect) takes place on the same time-scale as the SP motion. The main difference is that AEE is "controlinduced" effect, while the SP heavily damped oscillations are the result of airplane's inherent longitudinal stability. 

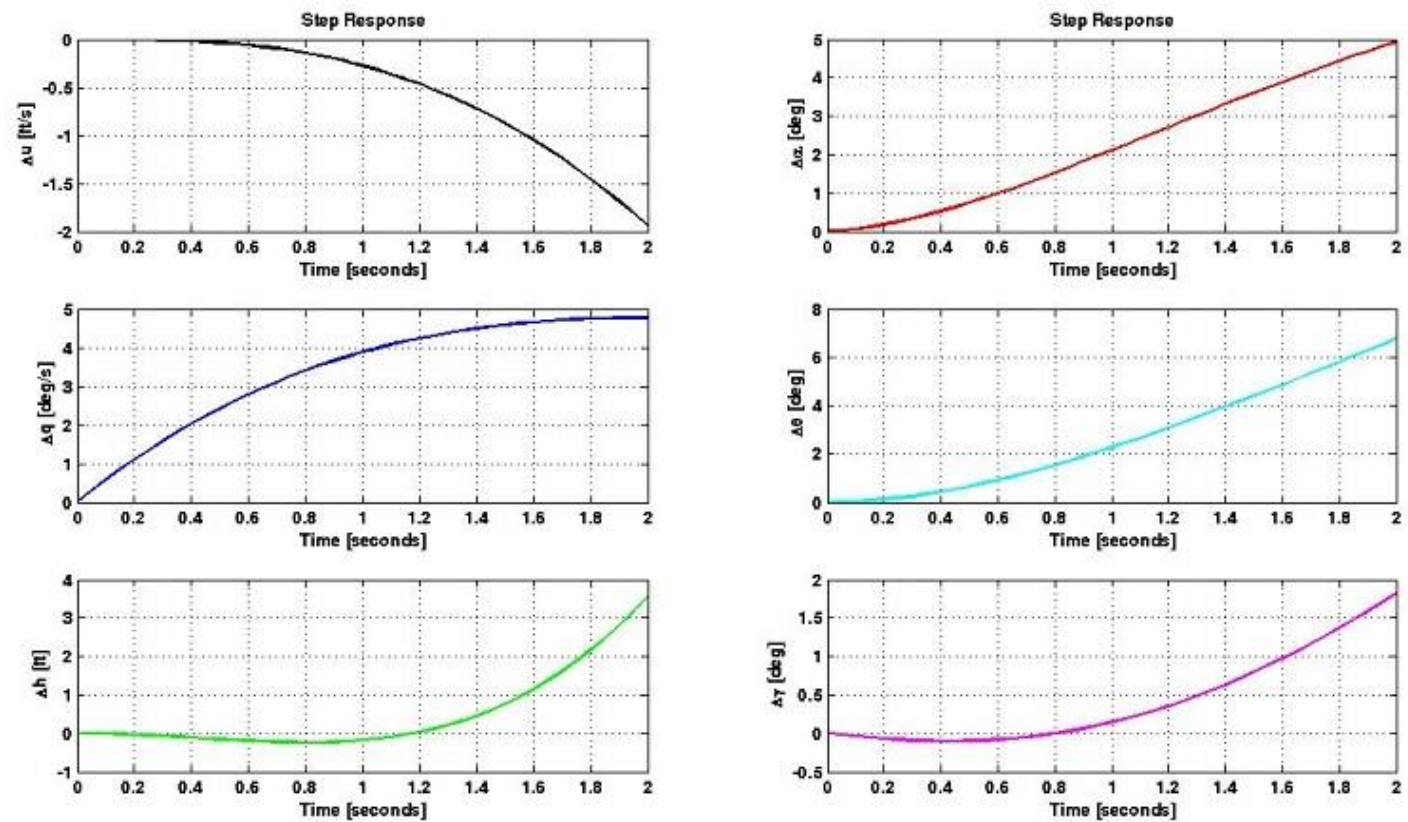

Figure 13. Unrestricted longitudinal dynamics flight perturbations due to step $10^{0}$ elevator up (-) control input using the full 3-DOF state-space model with kinematic relationships of free-flight for B747-200.

Clearly, the forward speed change in flare with no thrust inputs and for short periods is small (one knot deceleration after $1.86 \mathrm{~s}$ ). The pitch angle (\#4 graph in Figure 13) and the AOA (\#2) increased by about 2 degrees each after about 1 second in the pull-up maneuver indicating that the flight path angle change (\#6) is zero (as in pure-pitching mode). Obvious is also the initial loss of altitude $(0.2 \mathrm{ft})$ and steepening the approach $\left(-0.1^{0}\right.$ at $\left.0.5 \mathrm{~s}\right)$ inducing downward vertical acceleration and speed increments. While altitude loss and downward acceleration are not that significant, the real problem is in creating the dead-time (lag) during a flare with the time-scales of about 1.18 seconds for height loss and 0.80 seconds for vertical airspeed (flight-path angle) reversal. This is about the same result as was obtained in a simple analytical model developed here. This is to be compared to 1.263 and $0.73 \mathrm{~s}$ as calculated for the altitude-loss and the vertical speed reversal times for the same airplane and conditions. Thus all the assumptions made and the results obtained from the simple model are validated. It is also possible to add atmospheric disturbances (horizontal and vertical gusts) as well as ground effect aerodynamics to get very faithful airplane response provided all design data are known. 
To summarize, the all three important height parameters are initially reversed. This is the consequence of the traditional tail elevator actually being the "wrong" control initially and for short period. This effect makes consistent accurate landings and touchdowns very difficult. Interestingly, regardless of the elevator force magnitude, the reversal duration does not change for a particular airplane type. The worst AEE consequence thus is to introduce the dead-time or the time-delay in pitch response. The landing flare occurs on the time-scale of few seconds and 1-2 seconds time-delays are essential.

Armed with the better understanding of the airplane longitudinal dynamics during flare maneuver and especially the inevitable occurrence of the AEE, it is now investigated how this "adverse" phenomenon can be used to actually improve landing control and accuracy.

The new proposed landing technique, illustrated in Figures 6 and 14, would start with the flare about 10 feet lower than usual for a particular airplane type (say, $20 \mathrm{ft}$ instead of $30 \mathrm{ft}$ gear height) utilizing somewhat faster pull-up of perhaps 1.20-1.25 $\mathrm{g}$ which is still well below the landing stall margin of $1.69 \mathrm{~g}$. This will result in achieving the pitch angle of perhaps 7-8 degrees (from 2-3 degrees nose-up in stabilized approach), and then almost instantaneously followed by a push-over pulse for about $600-800 \mathrm{~ms}$ relax and expect main gear touchdown. The push-over maneuver will be initiated when the airplane is about 4-6 feet above the ground. The main gear touchdown would still occur with the pitch (deck) angle of 5+ degrees and the vertical touchdown speeds in the range of 100-200 fpm. The scatter of landing touchdowns is expected to be significantly smaller with more consistent landings and significant runway distance savings achieved. Such accurate touchdowns would be crucial for landings on contaminated runways and for LAHSO operations.

In the push-over maneuver a pilot is trying to accomplish two things:

- Reduce the rate-of-descent by relying on the short-term AEE's reversealtitude response and reduction in ROD.

- Reduce the pitch angle and set the airplane in the pitch down motion which is going to save few seconds in lowering the nose gear down after main-gear touchdown.

In order to estimate the reduction in ROD by push-over maneuver, the law of conservation of linear momentum is employed for the vertical axis only: 


$$
\frac{d K_{z}}{d t}=\sum F_{z} \quad \Rightarrow \quad \int_{K_{z 1}}^{K_{z 2}} d K_{z}=\frac{W}{g} \int_{v_{z 1}}^{v_{z 2}} d v_{z}=\int_{t_{1}}^{t_{2}}\left(\sum F_{z}\right) d t
$$

From where it follows:

$$
\frac{W}{g}\left(R O D_{2}-R O D_{1}\right)= \pm \Delta L_{t} \cdot \Delta t \quad \Rightarrow \quad \Delta R O D= \pm \frac{g \cdot \Delta L_{t}}{W} \Delta t= \pm P \Delta t
$$

For example, assuming the initial pull that reduced the approach ROD from 720 (12 fps) to $300 \mathrm{fpm}$ (5 fps) and airplane at the pitch angle of 8 degrees nose up while only about $5 \mathrm{ft}$ above the runway. A pilot then generates a 50,000 lb upward (push-over) vertical pulse on the tail (elevator down) of an aircraft weighing 550,000 lb lasting about $800 \mathrm{~ms}(0.8 \mathrm{~s})$ by pushing the control column forward and then neutralizing. Such push-over pulse will create maximum climb ROD of $141 \mathrm{fpm}(2.34 \mathrm{fps})$ that must be added to the descent rate of $300 \mathrm{fpm}$ resulting in the net ROD of $160 \mathrm{fpm}(2.66 \mathrm{fps})$. This must be timed appropriately so that the airplane is about touching down at that moment.

Simultaneously, the pitch angle of the aircraft will decrease according to the law of conservation of angular momentum:

$$
I_{y y} \frac{d^{2} \theta}{d t^{2}}=I_{y y} \frac{d q}{d t}= \pm \Delta M_{t}=\mp \Delta L_{t} \cdot \Delta l_{t}
$$

The pitch rate will change:

$$
\int_{0}^{q_{1}} d q=\frac{ \pm \Delta M_{t}}{I_{y y}} \int_{0}^{t} d t=\frac{ \pm \Delta M_{t}}{I_{y y}} \Delta t \Rightarrow q= \pm \frac{\Delta M_{t}}{I_{y y}} t= \pm R t
$$

For example, if a pilot applies forward pressure pulse for $800 \mathrm{~ms}$, creating downward pitching moment of, $50,000 \cdot 100=5,000,000 \mathrm{ft} \cdot \mathrm{lb}$, in an airplane with lateral-axis moment of inertia of $3.0 \cdot 10^{7} \mathrm{slug} \cdot \mathrm{ft}^{2}$, it will result in the final pitch rate of about $7.64 \mathrm{deg} / \mathrm{s}$. The total pitch angle change in $800 \mathrm{~ms}$ pulse will be about 3 degrees or final 5 degrees nose-up pitch attitude (from 8 degrees after pull-up) at main-gear touchdown:

$$
\frac{d \theta}{d t}=q(t) \quad \Rightarrow \quad \int_{0}^{\theta} d \theta=\Delta \theta=\int_{0}^{t} q(t) d t= \pm \frac{\Delta M_{t}}{2 I_{y y}} t^{2}= \pm R \frac{t^{2}}{2}
$$


This analysis neglects pitch stiffness and damping as well as changing aerodynamics in ground effect. However, as it was seen from the full model the difference is not large and the assumptions made are quite reasonable. Another added benefit to executing push-over maneuver when at appropriate height and just before touchdown is that the landing gear located behind the CG will be rotated away from the runway surface therefore minimizing vertical speed at the moment of touchdown. However, that only reduces the initial impact, but does not affect the vertical speed of CG. For example, if the landing gear is about 20 feet beyond the instantaneous point of rotation with the nose-down rotation of $6 \mathrm{deg} / \mathrm{s}$ at the time of touchdown, the vertical speed decreases by additional $126 \mathrm{fpm}$. Accordingly, the AEE and the rotation of the landing gear can both combined reduce the vertical touchdown impact by additional 200-300 fpm. A maneuver of short push-over pulse can be beneficial when within $1 \mathrm{~s}$ of touchdown and at the heights not greater than 4-6 feet. Detailed full flight simulator (FFS) study involving pilots of various experiences and in different aircraft will have to be conducted to validate the main proposal in this article. The remaining question is if the FFS flight models running in real-time have the accuracy and fidelity to properly simulate AEE.

Standard flare technique involving starting flare higher to account for the AEE-induced time-lag carries some negative implications. First, it is more difficult to judge the height and it also implies shallower round-out (flare) which is perhaps preferred by crew, but it consumes excessive horizontal distance. Letting the aircraft settle gently onto runway may be preferred by pilots and passengers, but it often leads to landing long increasing the probability of overrun, excessive subsequent wear and tear of brakes and needlessly overstressing the entire airplane structure during heavy braking and thrust-reversing. Building longer runways is clearly not an option due to excessive cost and pilots could get even more complacent and losing touchdown control discipline.

The new proposed landing technique, which was perhaps "discovered" accidentally by some pilots could not have been consistently reproduced as the main causes were never understood properly. Taking everything into account it is believed that this new proposed landing/touchdown technique can save about 5001,000 feet of runway in average in regular daily line-operations. It will be of course most beneficial when landing on short contaminated runways and with LAHSO procedures where touchdown precision and prompt lowering of the nose gear is paramount.

It has been said that some pilots operating venerable tri-jet Boeing B727s used the "pull-push" method as a standard landing technique. It somehow worked 
despite the fact that many wrong explanations were given to explain it. The B727's operating manual does not explicitly endorse such technique for normal landings. However, because all three engines are installed in the tail, the airplane's CG (and wing) was quite behind and the arm of the elevator was relatively short. As a consequence, the powerful tail down force would create significant downward acceleration while the pitch response was taking time due to relatively weak control pitching moments. Accordingly a push-over maneuver created significant upward acceleration (reduced ROD) which B727 pilots apparently used frequently. It is possible that pull-push technique was also used by some pilots flying older airplanes which were "tail-heavy" having short tail-toCG arm (B727, Hawker Siddeley Trident, Vickers-Armstrong VC10, BAC 1-11, DC9/MD80, etc.). An airplane that had many landing accidents due to late flare is MD11. Supersonic and hypersonic airplanes of the future will be especially prone to AEE and landing/touchdown problems.

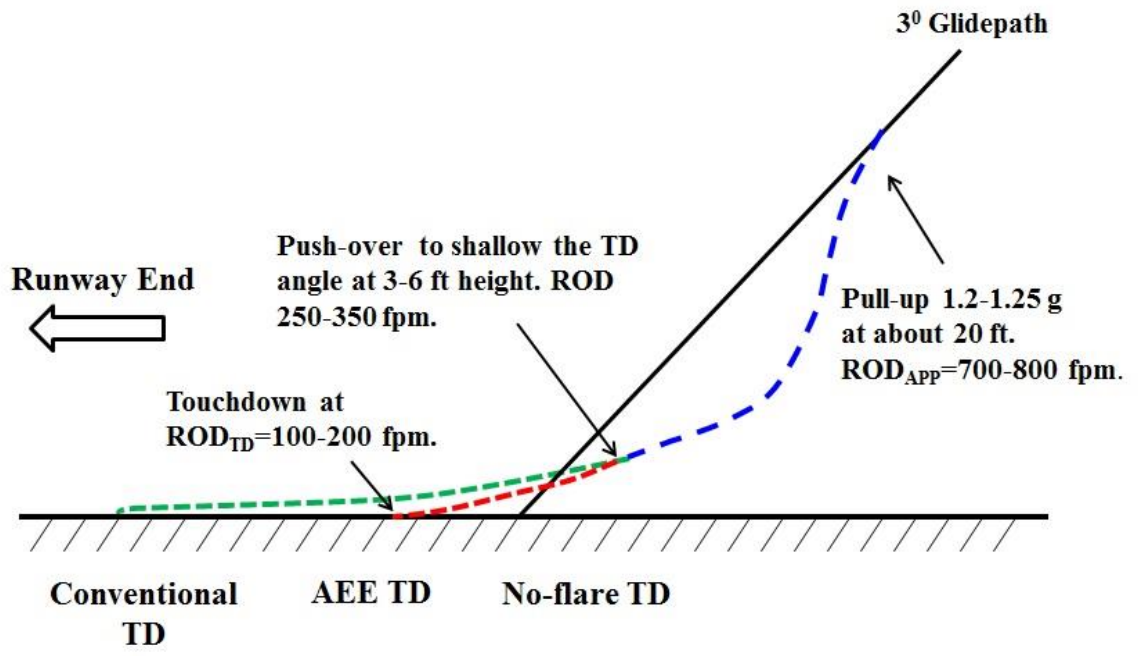

Figure 14. An illustration of the proposed new landing technique utilizing AEE. Not to scale. Angles and distances are highly exaggerated.

Although seemingly a peculiar phenomenon that could be dismissed as irrelevant, the AEE does exist every time an airplane with conventional tail is rotated for takeoff or in landing-flare pitch-up. There are few other lessons that can be learned by understanding the AEE and the short-term landing flare dynamics. When very close to ground with excessive ROD, a sudden pull-up to arrest the vertical descent rate could only make things worse and accelerate airplane downward even faster. At best there will be no effect of pitch-up correction as perhaps many pilots have already discovered in late flare (Daidzic, 
2010, 2011b). As much as it seems counter-intuitive, actually pushing over will rapidly create upward lift increment that will slow down descend. AEE is only a short-term effect and can be used only when close to ground, i.e., landing.

Since the pull-push landing technique involves two pitch rotations during which the glidepath is not significantly altered, an airplane utilizing this technique would essentially touch down very close (within 100-200 ft) to the minimum horizontal distance or where the glideslope/glidepath intercepts the runway. On the other hand, the "conventional" landing technique frequently results in touchdowns which are 500 to $1000+$ feet away from the glideslope runway intercept (no-flare touchdown) point. This is mostly due to the gradual flare and near-tangential touchdowns where the rounding slopes become too shallow. Such conventional landing technique causes large scattering in touchdown points. If two airplanes conducting "conventional" and "pull-push" landing enter the same gear-height at the same airspeed and ROD (glidepath), the normal landing technique would involve one rotation and slow gradual shallow descent toward the runway. The pull-push flare's two pitch rotations at lower altitudes actually involve maintaining the glidepath while the ROD decreases to acceptable 100-200 fpm touchdown.

More in-depth study and publications on AEE is planned in the near future. The ramp, parabolic, harmonic, rectified sine, finite pulse, doublet, and any arbitrary elevator control inputs will be used to investigate airplane's longitudinal dynamics. A full 6-DOF rigid-airplane nonlinear models and linearized coupled lateral-longitudinal small-perturbation airplane models will be used to model an arbitrary elevator pull-push landing flare maneuver with the complications of ground effect, atmospheric turbulence and gusty winds. It is also envisioned to conduct a piloted-study utilizing real airplanes and FFS to validate some of the theoretical results and develop the new landing technique for large airplanes. Optimization of the new pull-push landing technique and defining its envelope of use is of prime importance. Today's modern fly-by-wire airplanes utilize flare-mode for touchdown and that may appear to interfere with the "pullpush" technique explored here. However, if the new touchdown technique is found to be superior to the conventional one there should be no difficulty in modifying the flare-mode software and adapt to new realities. However, at this point we do not want to delve on too many speculations. Further research, flightpath integrations, and piloted studies will hopefully provide some more definite answers. 


\section{Conclusions}

A simple longitudinal flight dynamics model for rigid-body airplane landings was introduced. Only the short-period response to elevator up or down deflections were evaluated. Pitch stiffness, pitch damping, and vertical damping were neglected. The forward airspeed is essentially constant during the short term flare maneuver. Laplace integral transform was used to convert the differential model in time domain into the algebraic complex domain which after algebraic reductions, applications of control inputs, and solutions was then inverted back into the time domain. Only impulse and step control inputs were investigated in this study. Two different magnitudes of tail lift forces, corresponding to different elevator displacements were utilized to evaluate the effect on short-term height, AOA, vertical motion, and pitching dynamics. As expected, the simple model faithfully revealed the adverse elevator effect and the well-known reverse altitude response. A more complex mathematical models that were developed but not shown here due to complexity and size constraints validated and confirmed results of simple model used here. The AEE phenomenon was known for quite some time but often dismissed as irrelevant during flight at altitudes. However, during few seconds of landing flare and touchdown this adverse effect plays a prominent role which not only introduces initially reversed responses, but also generates substantial effective dead-times which are accounted for in operational practice by initiating round-out at higher altitudes and gentler than actually possible or desired. Today's standard manual landing and touchdown techniques actually introduce large scattering and significant uncertainties in touchdown accuracy. No flare touchdowns would be unacceptable due to severe landing gear structural loads. A new pull-push landing technique which utilizes AEE to pilot's advantage has been introduced and described. It is believed that the proposed landing and touchdown technique, in the absence of sophisticated direct-lift-control, would save, on average, 500 to 1,000 feet of runway which is especially important when landing on contaminated runways and during LAHSO operations. More results will be presented in subsequent publications involving full 6-DOF nonlinear and small-perturbation models while incorporating ground effect, atmospheric turbulence and wind. Experimental piloted studies in full-flight simulators, variable-stability, and real transport-category airplanes are required before the results and the proposed landing technique could be validated and perhaps implemented in daily operations. Experimental landing flare studies are difficult due to short duration of the maneuver. 


\section{Author Bios}

Dr. Nihad E. Daidzic is president of AAR Aerospace Consulting, L.L.C. He is also a full professor of Aviation, adjunct professor of Mechanical Engineering, and research graduate faculty at Minnesota State University. His Ph.D. is in fluid mechanics and Sc.D. in mechanical engineering. He was formerly a staff scientist at the National Center for Microgravity Research and the National Center for Space Exploration and Research at NASA Glenn Research Center in Cleveland, OH. He also held various faculty appointments at Vanderbilt University, University of Kansas, and Kent State University. His current research interest is in theoretical, experimental, and computational fluid dynamics, micro- and nano-fluidics, aircraft stability, control, and performance, mechanics of flight, piloting techniques, and aerospace propulsion. Dr. Daidzic is ATP and "Gold Seal" CFII/MEI/CFIG with flight experience in airplanes, helicopters, and gliders. 


\section{References}

Abzug, M. J., \& Larrabee, E. E. (1997). Airplane stability and control: A history of the technologies that made aviation possible. Cambridge, UK: Cambridge University Press.

Blake, W., \& Elliott, R. L. (1991, January). The last two minutes, Boeing Airliner, $1-9$.

Cleveland, F.A. (1970). Size Effects in Conventional aircraft Design, Journal of Aircraft, 7(6), 483-512

Daidzic, N. E., \& Shrestha, J. (2008). Airplane landing performance on contaminated runways in adverse conditions. Journal of Aircraft, 45, 2131-2144. doi: 10.2514/1.38056

Daidzic, N. E. (2009a, September). Avoiding veer-off accidents on contaminated runways, Professional Pilot, 43(9), 54-58.

Daidzic, N. E. (2009b, December). Avoiding overrun accidents on contaminated runways, Professional Pilot, 43(12), 104-107.

Daidzic, N. E. (2009c). Aircraft landing operations on contaminated runways, WATS 2009, World Aviation Training Symposium and Tradeshow, WATS Pilot Stream, Session 5: Air Carrier Training Insights, Rosen Shingle Creek Resort, Orlando, Florida, April 28-30, 2009.

Daidzic, N.E. (2010). Adverse elevator effect in landing flare, Paper WATS 5.3, WATS 2010, World Aviation Training Conference, Rosen Shingle Creek Resort, Orlando, Florida, April 27-29, 2010.

Daidzic, N. E. (2011a, March). Dealing with contaminated runways. The Journal for Civil Aviation Training (CAT), 2/2011, 29-32.

Daidzic, N. E. (2011b, May). Point of flare - the last five seconds, Professional Pilot, 45(5), 110-114.

Daidzic, N.E. (2011c) Some considerations for regional airline operations on contaminated runways, WATS 2011, World Aviation Training Conference, Rosen Shingle Creek Resort, Orlando, Florida, Paper RATS 6.3, April 19-21, 2011. 
Daidzic, N. E. (2013, August). T/O overruns and veer-offs on slippery runways with crosswinds, Professional Pilot, 47(8), 54-58.

Davis, D.P. (1971). Handling the Big Jets. 3rd ed., London, UK: Civil Aviation Authority.

Denton, J. (1993). Airline Pilot: A guide to good practices and techniques, Auckland, New Zealand: DFT Publishing.

van Es, G. W. H., van der Geest, P. J., Cheng, A., Hackler, L., Dillard, A. E. (2007). A study of normal operational landing performance on subsonic, civil, narrow-body jet aircraft during instrument landing system approaches (Final Report DOT/FAA/AR-07/7), Washington, DC: USDOT, FAA.

Etkin, B. (1959). Dynamics of flight: Stability and control. New York, NY: John Wiley \& Sons.

Etkin, B. (2005). Dynamics of atmospheric flight. Mineola, NY: Dover.

Grantham, W. D., Smith, P. M., Person, L. H. Jr., Meyer, R. T., \& Tingas, S. A. (1987). Piloted simulator study of allowable time delays in large-airplane response (NASA TP-2652), Hampton, VA: NASA Langley Research Center.

Kolk, R. W. (1961). Modern flight dynamics. Englewood Cliffs, NJ: PrenticeHall, Inc.

Lykken, L. O., \& Shah, N. M. (1972). Direct Lift Control for Improved Automatic Landing and Performance of Transport Aircraft, Journal of Aircraft, 9, 325-332.

Lowery, J. (2001). Professional Pilot, 2nd ed., Ames, IA: Iowa State University Press.

Malmquist, S., Vincenzi, D. A., Liu, D. (2014). Kinematic effects in large transport aircraft. Int. J. Aviation Aeronautics Aerospace, 1(3), 1-16.

Merat, R. (2008). Study of a Direct Lift Control System Based on the A380 Aircraft, (AIAA paper 2008-1432), 46 ${ }^{\text {th }}$ AIAA Aerospace Sciences Meeting and Exhibit, January 7-10 2008, Reno, Nevada. 
Nelson, R. C. (1998). Flight stability and automatic control. 2nd ed., New York, NY: McGraw-Hill.

Phillips, W. F. (2004). Mechanics of flight. New York, NY: John Wiley \& Sons.

Pinsker, W. J. G. (1969). The Landing Flare of Large Transport Aircraft (R. \& M. 3602), UK Ministry of Technology, Aeronautical Research Council, UK: Her Majesty's Stationery Office.

Phillips, W.H. (1979) Altitude response of several aircraft during landing approach (NASA TM 80186), Hampton, VA: NASA Langley Research Center.

Roskam, J. (2007). Airplane flight dynamics and automatic flight controls. Part I, Lawrence, KS: DAR Corporation.

Russell, J. B. (1996). Performance and stability of aircraft. London, UK: Arnold.

Schiff, B. (1997). The Proficient Pilot. Volume 3, Newcastle, WA: Aviation Supplies \& Academics, Inc.

Seckel, E. (1964). Stability and control of airplanes and helicopters. New York, NY: Academic Press.

Seckel, E., (1975) The Landing Flare: An Analysis and Flight-Test Investigation (NASA Contractor Report CR-2517), Princeton University for NASA Langley Research Center, Washington, DC: NASA

Spiegel, M. R. (1965). Laplace Transforms, New York, NY: McGraw-Hill.

Schmidt, D. K. (2012). Modern fight dynamics. New York, NY: McGraw-Hill.

Stengl, R. F. (2004). Flight dynamics. Princeton, NJ: Princeton University Press.

Stevens, B. L., \& Lewis, F. L. (2003). Aircraft control and simulation. 2nd ed., Hoboken, NJ: John Wiley \& Sons, Inc.

Stickle, J. W. (1961). An investigation of landing-contact conditions for two large turbojet transports and a turboprop transport during routine daylight operations (NASA TN D-899), Langley Field, VA: NASA Langley Research Center. 
US Department of Transportation, Federal Aviation Administration. (2013). Part 25, Airworthiness Standards: Transport Category Airplanes. Washington, DC: Author.

Webb, J., \& Walker, B. (2004). Fly the Wing. 3rd ed., Ames, IA: Blackwell Publishing.

White, M. D. (1968). Proposed analytical Model for the final stages of landing a transport airplane (NASA TN D-4438), Moffett Field, CA: NASA Ames Research Center.

Zaal P. M. T., Pool D. M., de Bruin, J., Mulder, M., \& van Paassen M. M. (2008). Pilot's use of pitch and heave motion cues in a pitch control task (AIAA paper 2008-6537), AIAA Modeling and Simulation Technologies Conference and Exhibit, August 18-21 2008, Honolulu, Hawaii. 